Justyna M. Torbiarczyk ${ }^{1 *}$, Patryk A. Sobczak ${ }^{2^{*}}$, Katarzyna K. Torbiarczyk ${ }^{2}$, Joanna Miłkowska-Dymanowska', Adam Antczak ${ }^{3}$, Paweł Górski ${ }^{1}$, Adam J. Białas ${ }^{1 *}$, Wojciech J. Piotrowski ${ }^{1 *}$

'Department of Pneumology and Allergy, $1^{\text {st }}$ Chair of Internal Medicine, Medical University of Lodz, Poland

${ }^{2}$ Student of Medical Division, Medical University of Łódź, Poland

${ }^{3}$ Department of General and Oncological Pulmonology, Medical University of Łódź

*equal contribution

\title{
Is bronchoscopy always justified in diagnosis of haemoptysis?
}

The authors declare no financial disclosure

\begin{abstract}
Introduction: Fiber-optic bronchoscopy (FOB) is commonly performed in the first line in diagnostic process of haemoptysis. However, lots of evidences suggest that in haemoptysis FOB do not always provide additional, useful information to radiological diagnostics. Therefore, this study aimed to evaluate the validity of performing FOB as a method of choice in first line diagnostics of haemoptysis.

Material and methods: Data comprised consecutively collected results from patients referred for FOB assessment for clinical purposes were retrospectively analysed. We included all examinations, which were performed due to haemoptysis as the only indication, excluding any other lung-related conditions.

Results: 114 patients were finally included to the study. The median age was 59 (IQR: 46-64.75). Active bleeding was visualized during examination of $13(11.4 \%)$ patients. Patients with active bleeding did not differ significantly according to age: 59 (54-69) vs 59 (45-64) years; $W=532.5$, $p$-value $=0.27$, and gender: $\chi 2=1.68$, $\mathrm{p}$-value $=0.2$. 0 n the other hand, in $29(25.44 \%)$ patients, FOB revealed no visible abnormalities. This subgroup of patients was significantly younger - 46 (34-62) years vs 60 (53-67) years; $W=782, p$-value $=0.003$.

Conclusion: Low number of visualized active bleeding sites suggest overuse of bronchoscopy in diagnosis of haemoptysis. Indications for this examination should be reconsidered especially in young patients with non-massive haemoptysis.
\end{abstract}

Key words: fiber-optic bronchoscopy, FOB, haemoptysis, diagnostic procedures

Adv Respir Med. 2018; 86: 13-16

\section{Introduction}

Haemoptysis is defined as the expectoration of blood originating from the tracheobronchial tree or pulmonary parenchyma [1]. It can be divided into non-massive and massive. Massive haemoptysis is a life-threatening condition because it may cause sudden airway or haemodynamic compromise [2]. The most common causes of haemoptysis in industrialised nations are bronchitis, bronchiectasis and bronchogenic carcinoma [3].

Once the airway protection and volume resuscitation are ensured, fiberoptic bronchoscopy
(FOB) plays a pivotal role with regard to localisation of the anatomic site of bleeding, isolation of the involved airway, control of haemorrhage and treatment of the underlying cause of haemoptysis in case of visible endoluminal lesions [4]. However, there is no consensus about the timing of its performance [5].

Strong evidence suggests that in haemoptysis, FOB does not always add additional, useful information to radiological diagnostics. Nielsen et al. [6] reported that combining FOB and computed tomography (CT) does not result in a significant increase in sensitivity in discovering malignant or

Address for correspondence: Wojciech J. Piotrowski, Department of Pneumology and Allergy, 1st Chair of Internal Medicine, Medical University of Lodz,

ul. Kopcinskiego 22, 90-153 Lodz, Poland, e-mail: wojciech.piotrowski@umed.lodz.pl

DOI: 10.5603/ARM.2018.0004

Received: 08.11.2017

Copyright (C) 2018 PTChP

ISSN 2451-4934 


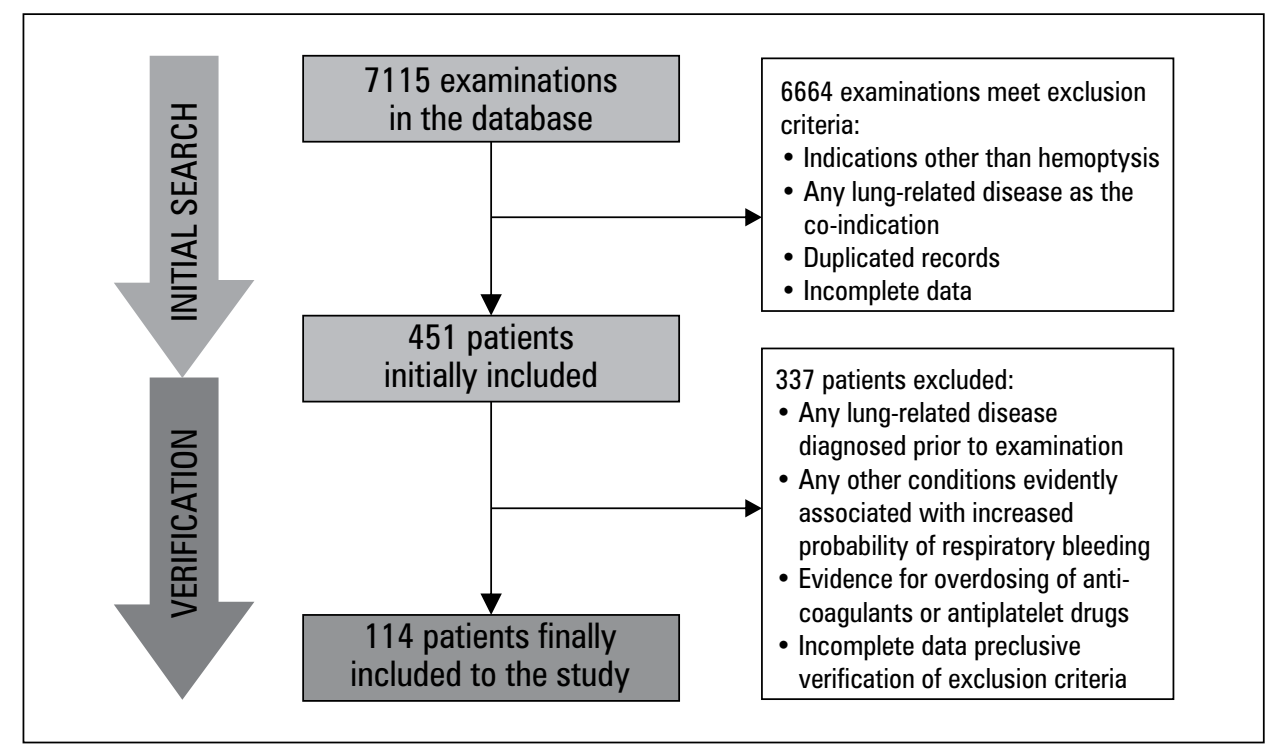

Figure 1. The flow chart of patient selection for inclusion in the study

non-malignant causes of haemoptysis [6]. Besides the obvious benefits associated with the visualisation of active bleeding and opportunity for treatment, the high number of performed FOBs may result in higher occurrence of potential complications such as hypoxaemia, haemodynamic variations, fever, infections, bronchospasm, pneumothorax, or other severe complications including death [7].

Therefore, the aim of the study was to evaluate the validity of performing FOB as a method of choice in first line diagnostics of haemoptysis. We hypothesise that the use of bronchoscopy in this context is often overused.

\section{Material and methods}

The study is observational, retrospective, single-site, based on patients admitted to the Bronchoscopy Unit of the Department of Pneumology and Allergy and Department of General and Oncological Pulmonology of Medical University of Lodz, suffered from haemoptysis. Only white Caucasians were included in the study. The studies were performed between November 2006 and April 2017 by respiratory physicians experienced in performing FOB. The results of performed examinations were routinely collected in digital database of the Bronchoscopy Unit. Conventional white light FOB was used in all patients.

Data comprising consecutively collected results from patients referred for FOB assessment for clinical purposes were retrospectively analysed. We included all examinations that were performed due to haemoptysis as the only indication. Patients with any previously diag- nosed lung-related disease were excluded (e.g. neoplasm, asthma, sarcoidosis). Any lesions significantly associated with haemoptysis found on chest radiogram were the exclusion criterion as well. We also excluded patients admitted to the department because of overdosing of anticoagulants or antiplatelet drugs, as well as with any other conditions evidently associated with increased probability of respiratory bleeding.

Continuous data were presented as the median with interquartile range (IQR). The U-Mann Whitney test was used, when data presented distribution other than normal. Categorical variables were expressed as an absolute value and percentage, and were compared using the Chi-square test. P-value $<0.05$ was considered as statistically significant.

Data were analysed using $\mathrm{R}$ software for MacOS (R Core Team (2016). R: A language and environment for statistical computing. R Foundation for Statistical Computing, Vienna, Austria).

\section{Results}

A total of 114 patients were finally included in the study - 63 (55.26\%) males and 51 (44.74\%) females, with the median age of 59 (46-64.75). Figure 1 presents the flow chart of patient selection for inclusion in the study.

In 29 (25.44\%) people, FOB revealed no visible abnormalities. Active bleeding, regardless its cause, was found during examination of $13(11.4 \%)$ patients (Fig. 2). The subjects with active bleeding did not differ significantly in terms of age: 59 (54-69) years vs $59(45-64)$ years; $\mathrm{W}=532.5$, $\mathrm{p}$-value $=0.27$, and gender: $\chi^{2}=1.68$, p-value $=0.2$. 


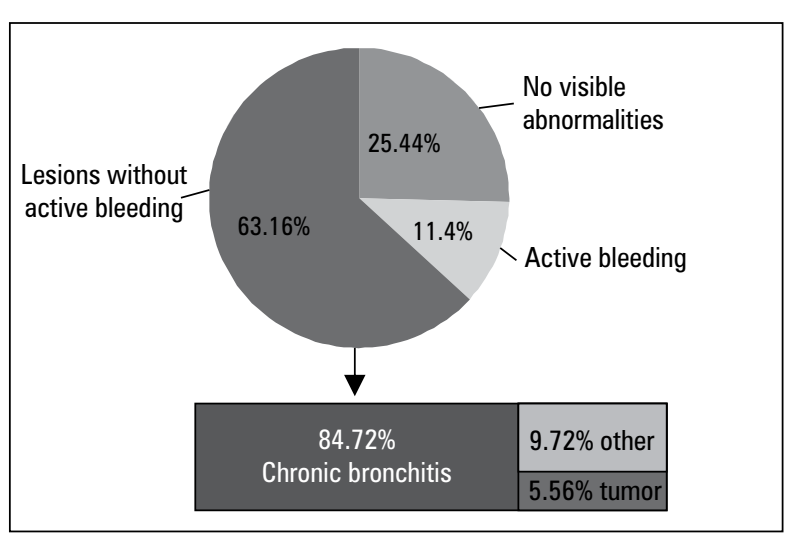

Figure 2. Endoscopic results of bronchoscopy

We also detected that patients with no visible abnormalities were significantly younger -46 (34-62) years vs 60 (53-67) years; $W=782$, p-value $=0.003$, however, we found no difference in respect of gender $\chi^{2}=1.71$, p-value $=0.19$.

In the rest of examinations, we observed lesions without active bleeding (63.16\%). In this group, FOB revealed: features characteristic for chronic bronchitis (61 patients; 84.72\%), intrabronchial tumor (4 patients; $5.56 \%$ ), or other unspecified lesions (7 patients; 9.72\%) (Fig. 2).

Our database revealed 4 patients with massive haemoptysis, what precluded further analyses of that subgroup.

\section{Discussion}

Our results showed a low number of visualised active bleeding (11.4\%). Also, we did not detect any significant difference between patients in terms of age and gender, when stratified by the visualisation of active bleeding.

In other diagnosed conditions such as bronchitis, neoplasms, or suspicion of diffused alveolar haemorrhage, the examination provided no additional, clinically useful information to radiological diagnostics. Moreover, often it had no impact on further proceedings.

Furthermore, in more than a quarter of patients, FOB did not reveal any pathological changes. We also observed that FOBs, in which we observed no visible lesions, were performed in significantly younger patients.

Presented results might arise from restrictions of FOB following the character, anatomical location and size of lesions. It has limited sensitivity in diagnosing distant bronchial and parenchymal abnormalities. However, it is noted that FOB is better in evaluating mucosal abnor- malities and providing material for pathological diagnosis [8].

In patients presenting haemoptysis, besides FOB, chest radiography and CT for primary screening are used. Many studies were performed to compare value of these examinations [8-13]. Davoodi et al. [9] compared diagnostic accuracy of multi-detector CT (MDCT) and FOB in haemoptysis, and reported that MDCT scan detected the site of bleeding more efficiently and had higher sensitivity in identifying the cause of haemoptysis. Likewise, Thirumaran et al. [8] concluded that even though CT and FOB are complementary, CT is preferable in detecting abnormalities that explain the cause of haemoptysis. Moreover, in non-massive haemoptysis, Gasparini recommended to perform bronchoscopy after CT scan [5]. According to previous studies, in many causes of haemoptysis, e.g. bronchiectasis, idiopathic pulmonary fibrosis (IPF) and neoplasm, FOB is inferior in identification of pathological lesions in comparison to CT $[8,9,14]$. In the context of bronchogenic carcinoma, Sharma et al. [13] concluded that in patients with normal chest radiogram and haemoptysis, FOB plays a minor role in the condition, especially when risk factors are absent. Moreover, there is an evidence that if chest CT does not find any pathological changes, FOB is most unlikely to reveal anything of significance [12].

According to previous investigations and our results, and taking into account the balance between the risk and benefits of the procedure, the role of FOB in the first line diagnostic process of haemoptysis ought to be reconsidered. Essentially, focused medical history and physical examination should help identify aetiologic clues [11], and subsequent procedures should be considered as additional tools for differential diagnosis.

There are several limitations of the study, that need to be considered. First of all, as a retrospective, single-institution study, it is burdened by all limitations associated with this type of data collection, including an absence of data regarding potential confounding factors. Moreover, we were not able to verify severity of haemoptysis, because in available digital records there is a lack of data about estimated amount of expectorated blood. This is why, we did not perform analysis taking into account the severity of bleeding. Moreover, our database comprises 4 patients with massive haemoptysis, what precluded further reliable analysis of that subgroup. Finally, due to retrospective type of analysis, we were not able to pro- 
vide complete imaging data to verify our results with the type and distribution of CT-detected abnormalities. To provide this information, we need a sufficiently powered prospective study on a well-described sample.

\section{Conclusion}

A low number of visualised active bleeding sites suggests overuse of bronchoscopy in diagnosis of haemoptysis. Indications for this examination should be reconsidered especially in young patients with non-massive haemoptysis.

\section{Acknowledgements}

The authors want to express their gratefulness for all employees of the Bronchoscopy Unit of the Department of Pneumology and Allergy and Department of General and Oncological Pulmonology of Medical University of Lodz for their great contribution to this study. In particular: Jerzy Marczak, Jadwiga Kroczyńska-Bednarek, Sebastian Majewski, Kamil Szyszow, Marek Zięba, Sylwia Kryczkowska-Krawczyk and Anna Lewandowska. Authors want also to thank Mr. Józef Sobczak for his contribution to this study.

\section{Conflict of interest}

Authors declare no conflicts of interests.

\section{References:}

1. Jeudy J, Khan AR, Mohammed TL, et al. Expert Panel on Thoracic Imaging. ACR Appropriateness Criteria hemoptysis. J Thorac Imaging. 2010; 25(3): W67-W69, doi: 10.1097/RTI.0b013e3181e35b0c, indexed in Pubmed: 20711032.

2. Ayed A. Pulmonary resection for massive hemoptysis of benign etiology. Eur J Cardiothorac Surg. 2003; 24(5): 689-693, indexed in Pubmed: 14583299.

3. Dugosh JW. Ph D. Hemoptysis. Salem Press Encycl Health. 2017.

4. Sakr L, Dutau H. Massive hemoptysis: an update on the role of bronchoscopy in diagnosis and management. Respiration. 2010; 80(1): 38-58, doi: 10.1159/000274492, indexed in Pubmed: 20090288.

5. Gasparini S. Indications for diagnostic bronchoscopy in adults. Monaldi Arch Chest Dis. 2011; 75(1): 24-31, doi: 10.4081/ monaldi.2011.236, indexed in Pubmed: 21626989.

6. Nielsen K, Gottlieb M, Colella S, et al. Bronchoscopy as a supplement to computed tomography in patients with haemoptysis may be unnecessary. Eur Clin Respir J. 2016; 3: 31802, indexed in Pubmed: 27343164.

7. Leiten EO, Martinsen EM, Bakke PS, et al. Complications and discomfort of bronchoscopy: a systematic review. Eur Clin Respir J. 2016; 3: 33324, indexed in Pubmed: 27839531.

8. Thirumaran M, Sundar R, Sutcliffe IM, et al. Is investigation of patients with haemoptysis and normal chest radiograph justified? Thorax. 2009; 64(10): 854-856, doi: 10.1136/ thx.2008.108795, indexed in Pubmed: 19457872.

9. Davoodi M, Kordi M, Gharibvand MM, et al. Hemoptysis: comparison of diagnostic accuracy of multi detector CT scan and bronchoscopy. Glob J Health Sci. 2015; 7(3): 373-377, doi: 10.5539/gjhs.v7n3p373, indexed in Pubmed: 25948463.

10. Tsoumakidou M, Chrysofakis G, Tsiligianni I, et al. A prospective analysis of 184 hemoptysis cases: diagnostic impact of chest X-ray, computed tomography, bronchoscopy. Respiration. 2006; 73(6) 808-814, doi: 10.1159/000091189, indexed in Pubmed: 16446530.

11. Earwood JS, Thompson TD. Hemoptysis: evaluation and management. Am Fam Physician. 2015; 91(4): 243-249, indexed in Pubmed: 25955625.

12. Bønløkke S, Guldbrandt LM, Rasmussen TR. Bronchoscopy in patients with haemoptysis and normal computed tomography of the chest is unlikely to result in significant findings. Dan Med J. 2015; 62(8): A5123, indexed in Pubmed: 26239592.

13. Sharma SK, Dey AB, Pande JN, et al. Fiberoptic bronchoscopy in patients with haemoptysis and normal chest roentgenograms. Indian J Chest Dis Allied Sci. 1991; 33(1): 15-18, indexed in Pubmed: 1791016.

14. Set PA, Flower CD, Smith IE, et al. Hemoptysis: comparative study of the role of CT and fiberoptic bronchoscopy. Radiology. 1993; 189(3): 677-680, doi: 10.1148/radiology.189.3.8234690, indexed in Pubmed: 8234690. 